\title{
FITOPLÂNCTON COMO FERRAMENTA DE GESTÃO AMBIENTAL NA PRAIA DE BRASÍLIA TEIMOSA, PERNAMBUCO, NORDESTE DO BRASIL.
}

Recebido em: 08/01/2013

Aceito em: 25/10/2013

\author{
Leandro Cabanez FERREIRA ${ }^{1}$ \\ Maria da G. G. da SILVA CUNHA ${ }^{1}$ \\ Alessandra Lee B. FIRMO ${ }^{2}$ \\ Gislayne Cristina Palmeira BORGES ${ }^{1}$ \\ Jucicleide Cabral de LIMA $^{1}$ \\ Emersom de Paula LIMA ${ }^{1}$ \\ Nayana Burque Antão da SILVA ${ }^{1}$
}

RESUMO

\begin{abstract}
O objetivo deste trabalho foi avaliar os parâmetros ambientais, biomassa, comunidade fitoplanctônica e espécies bioindicadoras, como ferramenta de gestão ambiental na praia Brasília Teimosa, Pernambuco ( $\left.8^{\circ} 04^{\prime} \mathrm{S} ; 3^{\circ} 52^{\prime} \mathrm{W}\right)$. Parâmetros abióticos (precipitação, temperatura da água, salinidade, material em suspensão, teor e taxa de saturação do oxigênio, nitrito, nitrato, fosfato e silicato) foram obtidos simultaneamente às coletas de fitoplâncton (garrafa e rede) no período chuvoso (maio; junho e julho/2005) e de estiagem (novembro e dezembro/2005; janeiro/2006). Com exceção dos valores de nitrato (maiores no período chuvoso), os demais parâmetros estiveram dentro dos padrões recomendados. Foram identificados 84 taxa,
\end{abstract}

com maior representatividade das diatomáceas em abundância e frequência, Asterionellopsis glacialis, Chaetoceros lorenzianus, Cylindrotheca closterium, Helicotheca tamesis, Licmophora abbreviata e Skeletonema costatum. Altos níveis de nitrato, clorofila $a$, número de células por litro e espécies bioindicadores de eutrofização (Oscillatoria sp., Scenedesmus sp., Euglena sp., Synedra sp., Pleurosigma sp., Pseudo-nitzschia sp. e Skeletonema costatum) corroboraram a influência das atividades humanas na área, evidenciando um possível comprometimento da sanidade ambiental relacionado ao período de estiagem (período de maior exploração turística-recreativa).

Palavras-chave: Bioindicadores, Clorofila $a$, Florescimentos, Diatomáceas.

\section{ABSTRACT}

This study aimed to make a comparative analysis of environmental parameter variation, biomass variation, phytoplankton community structure and to identify water quality bioindicators species from Brasília Teimosa beach $\left(8^{\circ} 04^{\prime} \mathrm{S}\right.$; $34^{\circ} 52^{\prime} \mathrm{W}$ ), in Pernambuco coast, Brazil Northeast. Bottle and net samples were collected during the rainy (May, June and July/2005) and dry seasons (November, December/2005 and January/2006) with simultaneous environmental data collection (water temperature, salinity, suspended material, dissolved oxygen content and saturation rate, nitrite, nitrate, phosphate, silicate and rainfall). Results showed that, except only for nitrate (with higher values during the rainy season), the environmental parameters were within the recommended limits. Among the 84 infrageneric taxa identified, diatoms were the most representative group regarding abundance and frequency, with Asterionellopsis glacialis (Castracane) Round, Chaetoceros lorenzianus Grunow, Cylindrotheca closterium (Ehrenberg) Reiman \& Lewis, Helicotheca tamesis (Shrubsole) Ricard, Licmophora abbreviata Agardh e Skeletonema costatum (Greville) Cleve. High levels of nitrate, chlorophyll-a, number of cells per liter and eutrophication's bioindicators species (Oscillatoria sp., Scenedesmus sp., Euglena sp., Synedra sp., Pleurosigma sp., Pseudo-nitzschia sp. and Skeletonema costatum) confirmed the influence of human activities in the area, indicating a possible commitment of

1 - Universidade Federal de Pernambuco, Departamento de Oceanografia, Laboratório de Fitoplâncton,

Cidade Universitária, Av. Arquitetura s/n, Recife, PE, CEP 50-740-550, Brasil.

2 - Instituto Federal de Educação, Ciência e Tecnologia de Pernambuco - IFPE

Contatos: leocabanez@yahoo.com.br; carlosegloria@uol.com.br; alessandra.lee@gmail.com; 
FERREIRA, L. C. et al. Fitoplâncton como ferramenta de gestão ambiental na praia de Brasília Teimosa, Pernambuco, Nordeste do Brasil.

environmental health related to dry season

(period of greatest tourist/recreational use).

Key Words: Bioindicators, Chlorophyll a, Blooms, Diatoms.

\section{INTRODUÇÃO}

Perturbações antrópicas como poluição orgânica, industrial e construções à beira mar, vêm afetando os ecossistemas marinhos, causando visíveis modificações no desenvolvimento, sobrevivência e dispersão dos organismos, selecionando uma biota adaptada com complexos padrões de distribuição espacial e temporal (RÖRIG et al., 2006).

Queiroz (2002) cita que a presença de determinados organismos está estritamente associada às condições ambientais específicas e que a avaliação dessas condições por meio de bioindicadores deverá tornar-se um procedimento fundamental para a administração, exploração racional e proteção dos ecossistemas aquáticos.

O fitoplâncton, assim como outros microrganismos, é utilizado para diagnosticar o ambiente aquático, devido a uma alta taxa de crescimento por unidade de tempo, desenvolvendo mecanismos para otimizar suas respostas fisiológicas às variações ambientais que repercutem na estrutura e funcionamento da comunidade.

Em vista disto, a inclusão do estudo do fitoplâncton em projetos de monitoramento de áreas marinhas costeiras ou oceânicas pode resultar em uma grande contribuição, uma vez que estas espécies, por responderem rapidamente aos impactos ambientais, representam bons indicadores na identificação de possíveis modificações nas características ecológicas de áreas sujeitas à introdução de substâncias alógenas.

A praia de Brasília Teimosa, situada na região metropolitana do Recife, sofre a influência das diversas atividades que são desenvolvidas em seu entorno, e que refletem nas variações dos parâmetros físicos, químicos e biológicos da água, alterando a composição das comunidades aquáticas, bem como a vida da população humana dependente da mesma.

Neste contexto, é necessário um estudo detalhado (físico-químico-biológico) com objetivo de efetuar uma análise da estrutura da comunidade fitoplanctônica, variação da biomassa (clorofila a) e dos parâmetros ambientais, durante o período chuvoso e de estiagem, na praia de Brasília Teimosa, localizada em Pernambuco, Nordeste do Brasil.

\section{ÁREA DE ESTUDO}

O bairro de Brasília Teimosa surgiu na década de 60, da ocupação de uma área denominada Areal Novo, quando o então Presidente da República Juscelino Kubitschek construía Brasília-DF (RABELO, 1968). Os barracos eram erguidos à noite e, durante o dia, destruídos pela polícia, sempre em maior número, até que o lugar fez jus à teimosia dos ocupantes, denominando-se Brasília Teimosa. O bairro é caracterizado por sua referência de resistência popular: primeira invasão urbana do Brasil (BRANCO, 2004).

Está situado na orla marítima da zona sul da cidade, entre os bairros do Pina, Boa Viagem e área do Porto do Recife. Limita-se a leste com o oceano Atlântico; o rio Tejipió (Bacia do Pina) a noroeste; a Avenida Antônio de Góes a sudoeste; formando um triangulo com o vértice visando o Norte e se prolongando por uma fina linha de recifes que limitam o Porto (com aproximadamente $50 \mathrm{ha}$ ). A área é predominantemente plana com uma média de $2 \mathrm{~m}$ acima do nível da maré máxima. Possui uma população em torno de 30.000 habitantes (NUNES et al., 2005).

Por ter sido formada clandestinamente, sua orla possuía assentamentos informais que ocupavam de forma desordenada a área pública, com habitações em condições de risco físico e de insalubridade, as palafitas. Essa denominação refere-se ao conjunto de casas de madeira construídas em cima de estacas fincadas dentro do mar. A precariedade das condições sanitárias aliadas à falta de acesso ao local anulava o serviço de saúde preventiva, educacional e social. Foi necessária uma intervenção urbanística que visasse a retirada das palafitas e a relocação das famílias para garantir condições de segurança, protegendo-as contra o avanço do mar e melhorando a qualidade de vida da comunidade, que possui ligação muito forte com o 
FERREIRA, L. C. et al. Fitoplâncton como ferramenta de gestão ambiental na praia de Brasília Teimosa, Pernambuco, Nordeste do Brasil.

mar.

Com a construção da Avenida Brasília Formosa, na beira-mar, o bairro está revitalizado, transformado num futuro ponto de exploração imobiliária. Antes havia palafitas, agora existe uma avenida litorânea com calçadão, parques e quiosques. O local também abriga vários restaurantes de frutos do mar, além de ser a via de acesso para o dique onde fica o parque de esculturas de Brennand e o centro cultural do Antigo Recife.

Por enquanto, o mercado imobiliário não pode investir na nova orla, pois a área é classificada como Zona Especial de Interesse Social (ZEIS), que tem uma série de regras específicas que limitam o gabarito dos terrenos.

Caracteriza-se por apresentar um clima tipo Ams', ou seja, tropical quente e úmido, segundo o sistema Köppen de classificação, com uma precipitação pluviométrica anual que atinge mais de $2000 \mathrm{~mm}$. Existem pelo menos seis sistemas atmosféricos que produzem precipitação significativa na região: a zona de convergência intertropical (ZCIT), as bandas de nebulosidade associadas às frentes frias, os distúrbios de leste, os vórtices ciclônicos de ar superior (VCAS), as brisas terrestres e marítimas e as oscilações 30-60 dias. Esses fenômenos atuam em sub-regiões distintas (ARAGÃO, 2004).

Localizada no oceano Atlântico Sul, a área sofre ação da Corrente do Brasil, um ramo sul da Corrente Sul Equatorial. Junto à linha de praia atuam ainda as correntes longitudinais, correntes de retorno, correntes geradas por ondas e pelas marés. As longitudinais são as mais importantes, movendo-se paralelo à linha da costa, com velocidade que varia de acordo com o ângulo de incidência das ondas. As correntes geradas pelas ondas estão relacionadas com o movimento antepraia - costa-afora (e vice-versa), variando com o local e o clima de onda (ROLLNIC; MEDEIROS, 2006).

As marés do litoral Pernambucano são do tipo semidiurna, com período médio de 12,42 horas, apresentando duas preamares e duas baixa-mares por dia lunar; os valores representativos da altura da maré são de $2,4 \mathrm{~m}$ para sizígia máxima; $2,1 \mathrm{~m}$ para quadratura máxima. Ao longo da linha da costa metropolitana do Recife, as praias são compostas de areia quartzosa, onde em alguns lugares afloram as linhas de recifes e a pequena distância da costa pode ocorrer outras linhas de recifes que afloram apenas durante a baixa-mar (COUTINHO et al., 1997).

\section{MATERIAL E MÉTODOS}

Foi estabelecido um ponto de coleta situado em frente ao Iate Clube em Brasília Formosa sob as coordenadas $8^{\circ} 04^{\prime} 48^{\prime \prime} \mathrm{S}$ e $34^{\circ} 52^{\prime} 35^{\prime \prime} \mathrm{W}$ (Fig. 1), onde foram efetuadas seis coletas no total; compreendendo três meses no período chuvoso (maio, junho e julho de 2005) e três meses no período de estiagem (novembro e dezembro de 2005; janeiro de 2006), na praia de Brasília Teimosa, durante a preamar e baixa-mar de um mesmo dia.

Os dados climatológicos de precipitação pluviométrica foram procedentes do Instituto Nacional de Meteorologia (INMET), referente ao período estudo.

As coletas destinadas à análise qualitativa foram realizadas através de arrastos horizontais superficiais com auxílio de rede de plâncton com abertura de malha de $45 \mu \mathrm{m}$, durante cinco minutos. Posteriormente ao arrasto, o material foi fixado com formol neutro a $4 \%$, segundo recomendações de Newell e Newell (1963) e analisadas utilizando lâmina semipermanentes.

Para a análise quantitativa, amostras foram obtidas com auxílio de garrafa oceanográfica do tipo Kitahara, sendo posteriormente analisadas pelo método de Utermöhl (1958) em microscópio invertido WILD M-40 (resultados expressos em número de células por litro) (FERRARIO et al., 1995; HASLE, 1978).

Medidas de temperatura e salinidade da água foram obtidas in situ, respectivamente, com termômetro e refratômetro manual. 
FERREIRA, L. C. et al. Fitoplâncton como ferramenta de gestão ambiental na praia de Brasília Teimosa, Pernambuco, Nordeste do Brasil.

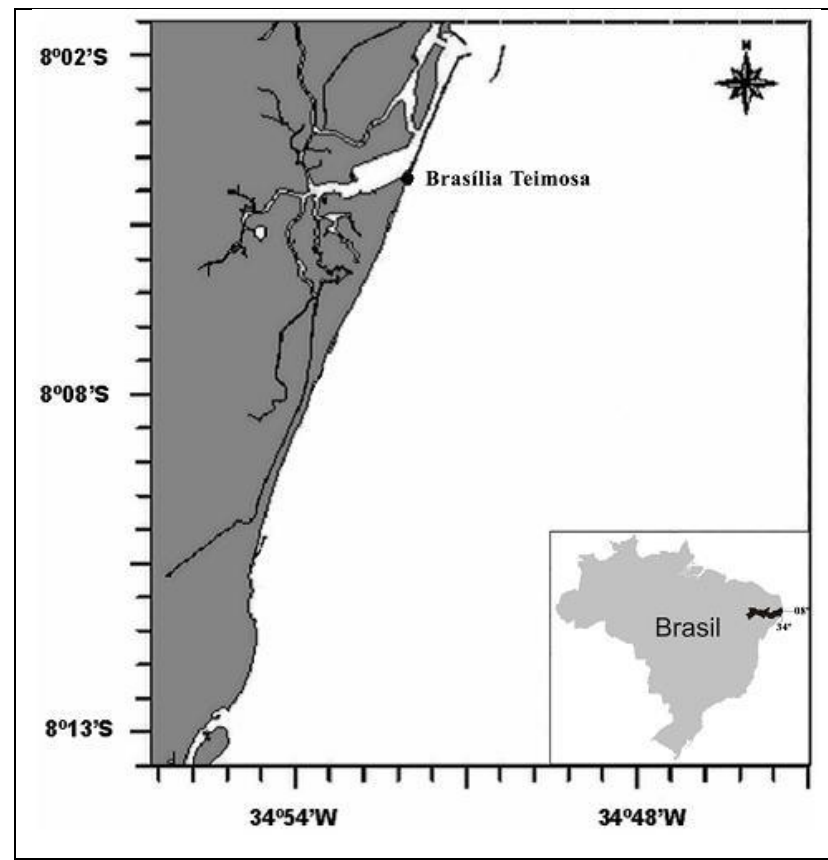

Figura 1 - Mapa da área de estudo com respectivo ponto de coleta.

As amostras utilizadas nas análises das variáveis hidrobiológicas $(\mathrm{pH}$, teor de oxigênio dissolvido, taxa de saturação do oxigênio, material particulado em suspensão, nitrito, nitrato, fosfato, silicato e clorofila a) foram coletadas com garrafa de Kitahara e seguidas as recomendações descritas em Strickland e Parsons (1972), UNESCO (1973), Melo et al. (1975) e Grasshoff et al. (1983).

Para interpretação da abundância relativa foram utilizadas as categorias dominantes para valores acima de $50 \%$ e abundantes para os valores superiores à média (LOBO; LEIGHTON, 1986). Em função do valor da frequência, os táxons foram classificados em ocorrência muito frequente $(>70 \%)$, frequente $(70 \mid-40 \%)$, pouco frequente $(40 \mid-10 \%)$ e esporádica $(\leq 10 \%)$.

O índice de Diversidade específica $\left(H^{\prime}\right)$ foi estimado segundo Shannon (1948), onde os resultados foram classificados em alta diversidade $\left(5 \mid-2,5\right.$ bits.Cel $\left.^{-1}\right)$, baixa diversidade $\left(2,5 \mid-1\right.$ bit.Cel $\left.^{-1}\right)$ e diversidade muito baixa ( $\leq 1$ bit.Cel $\left.^{-1}\right)$ (MARGALEF, 1978).

Foi efetuada uma análise de variância utilizando-se o teste não paramétrico de MannWhitney visando avaliar cada parâmetro estudado em função da sazonalidade e espacialidade, sendo considerados significativos os valores $\mathrm{p}<0,05$.

\section{RESULTADOS}

\section{Parâmetros Ambientais:}

A precipitação pluviométrica mensal acumulada referente aos anos de 2005 e 2006 apresentou uma média de $177,62 \mathrm{~mm}$, oscilando com um mínimo de $10,50 \mathrm{~mm}$, em janeiro de 2006 e um máximo de 490,00mm de chuva, em julho de 2005 (Fig. 2). 
FERREIRA, L. C. et al. Fitoplâncton como ferramenta de gestão ambiental na praia de Brasília Teimosa, Pernambuco, Nordeste do Brasil.

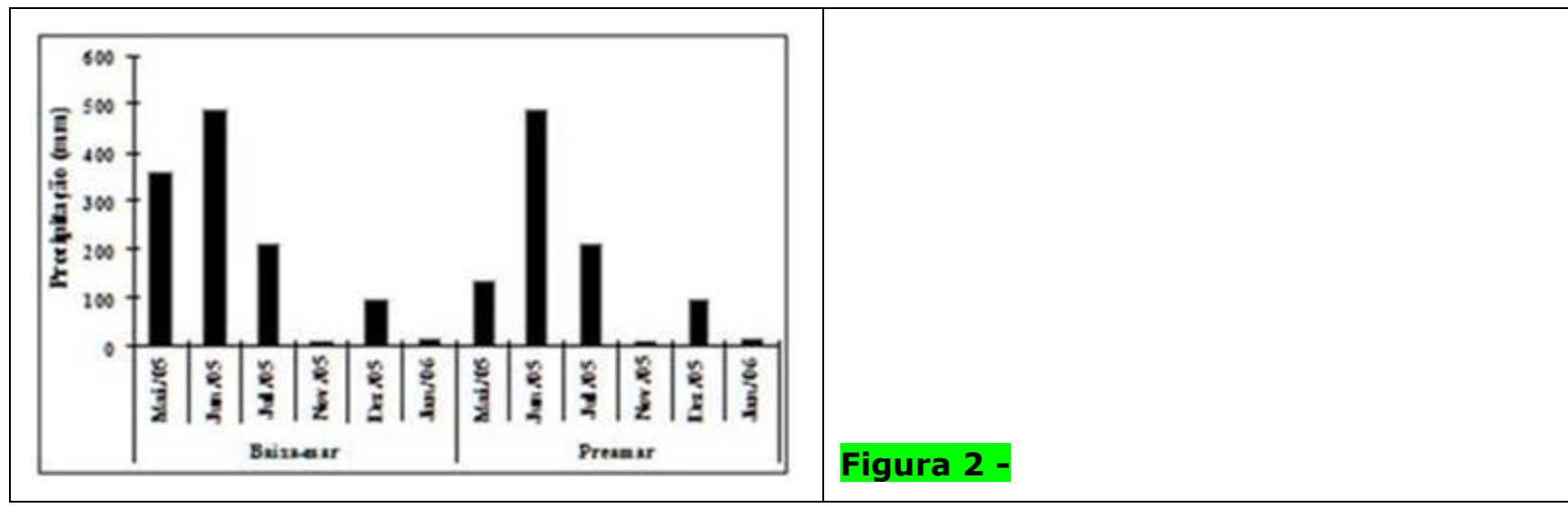

O valor médio da temperatura da água registrado durante o período de estudo foi de $29,40^{\circ} \mathrm{C}$, com uma amplitude térmica de $3,30^{\circ} \mathrm{C}$. Observou-se uma variação de 27,90 a $31,20^{\circ} \mathrm{C}$, sendo o valor mínimo encontrado na baixa-mar de jul./05, e o máximo na preamar de dez./05. A salinidade apresentou um valor médio de 33,25, com amplitude de 9 . 0 menor valor foi registrado em jun./05 (28), enquanto o maior, em jan./06 (37), ambos na preamar (Fig. 3a). Os valores do $\mathrm{pH}$ foram classificados como alcalinos, apresentando um valor médio de 8,47 e uma amplitude da ordem de 0,45 . Sua variação oscilou entre 8,23 e 8,68 , onde 0 menor valor foi registrado na baixa-mar em nov./05 e o máximo na preamar de dez./05 (Fig. 3b). As concentrações do oxigênio dissolvido registradas durante o período de estudo variaram de 4,95 a $6,91 \mathrm{ml}^{-\mathrm{L}^{-1}}$, sendo o valor mínimo encontrado na preamar de jul./05 e o valor máximo, em maio/05, também na preamar. A amplitude foi de $1,96 \mathrm{ml}^{-L^{-1}}$ e a média de $5,82 \mathrm{ml} . \mathrm{L}^{-1}$. O ambiente foi classificado como supersaturado durante o período de estudo (Fig. $3 c)$. Não houve diferenças significativas $(p>0,05)$ entre as marés e o período sazonal, para os parâmetros supracitados.

As concentrações do material em suspensão na água variaram de 30 a $96 \mathrm{mg}^{\circ} \mathrm{L}^{-1}$, sendo o mínimo encontrado na baixa-mar de nov./05 e máximo, na preamar de jul./05. A amplitude atingiu um valor de $66 \mathrm{mg} \cdot \mathrm{L}^{-1}$ e a média, $53,4 \mathrm{mg} \cdot \mathrm{L}^{-1}$ (Fig. $3 \mathrm{~d}$ ). Entre os períodos sazonais, os valores deste parâmetro foram significativamente diferentes $(p=0,03)$, com maiores valores no período chuvoso.

Para os sais nutrientes, o valor médio da concentração de nitrito registrado durante o período de estudo foi de $0,33 \mu \mathrm{mol} . \mathrm{L}^{-1}$, com uma amplitude de $0,72 \mu \mathrm{mol} . \mathrm{L}^{-1}$. As concentrações

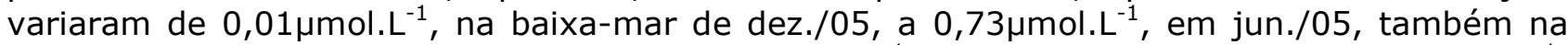
baixa-mar. A média do íon nitrato foi de $7,91 \mu \mathrm{mol} . \mathrm{L}^{-1}$, com uma amplitude de $21,35 \mu \mathrm{mol} . \mathrm{L}^{-1}$. As concentrações variaram de $0,09 \mu \mathrm{mol}^{-1} \mathrm{~L}^{-1}$ a $21,44 \mu \mathrm{mol} . \mathrm{L}^{-1}$, nas preamares dos meses de jan./06 e jun./05, respectivamente (Fig. 3e). Para o íon nitrato, foi possível observar uma diferença significativa $(p=0,00)$, onde os maiores valores no período chuvoso. O fosfato obteve média de $0,41 \mu \mathrm{mol} . \mathrm{L}^{-1}$, com uma amplitude de $0,96 \mu \mathrm{mol} . \mathrm{L}^{-1}$. As concentrações variaram de $0,03 \mu \mathrm{mol} . \mathrm{L}^{-1}$, na baixa-mar de jul./05, a $0,99 \mu \mathrm{mol} . \mathrm{L}^{-1}$, na preamar de jun./05. A concentração média de silicato foi de $18,68 \mu \mathrm{mol} . \mathrm{L}^{-1}$, amplitude de $34,91 \mu \mathrm{mol} . \mathrm{L}^{-1}$. Os valores variaram entre $6,25 \mu \mathrm{mol} . \mathrm{L}^{-1}$ e $41,16 \mu \mathrm{mol} . \mathrm{L}^{-1}$, encontrados na preamar de nov./05 e baixa-mar de jun./05, respectivamente (Fig. 3f). Não foi possível observar diferenças significativas $(p>0,05)$ para esses parâmetros. 

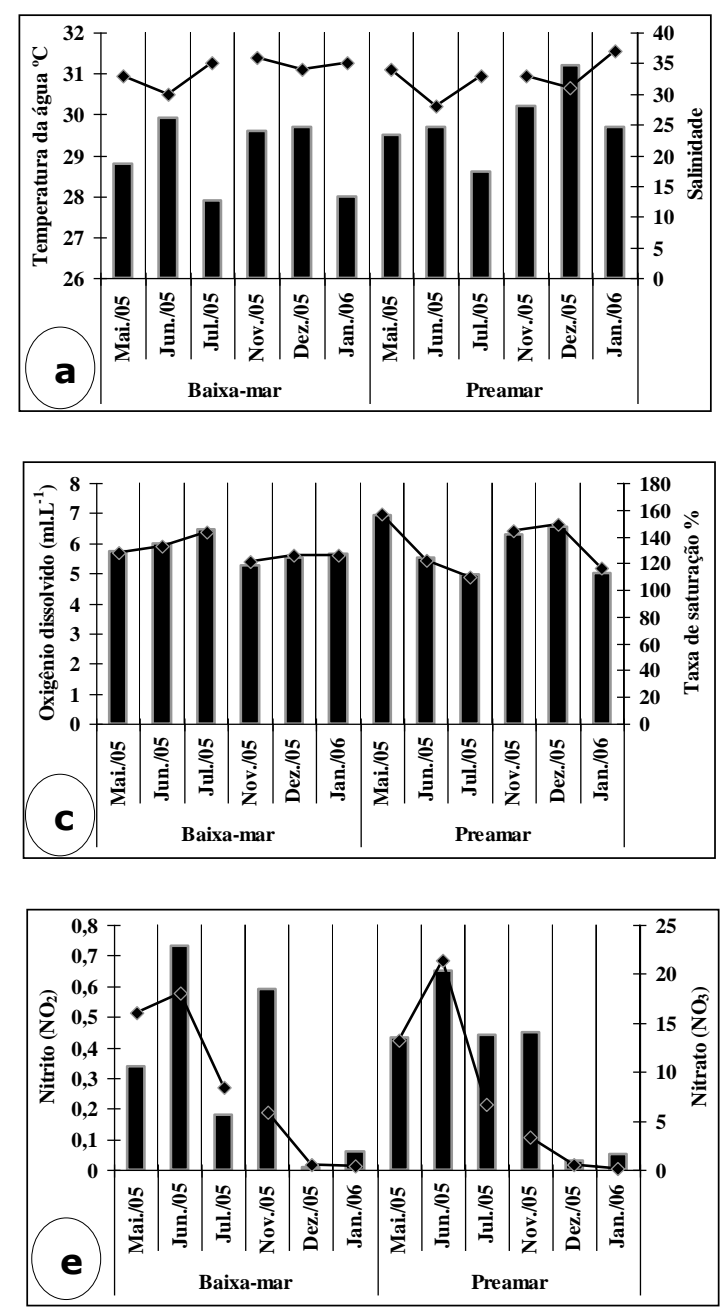
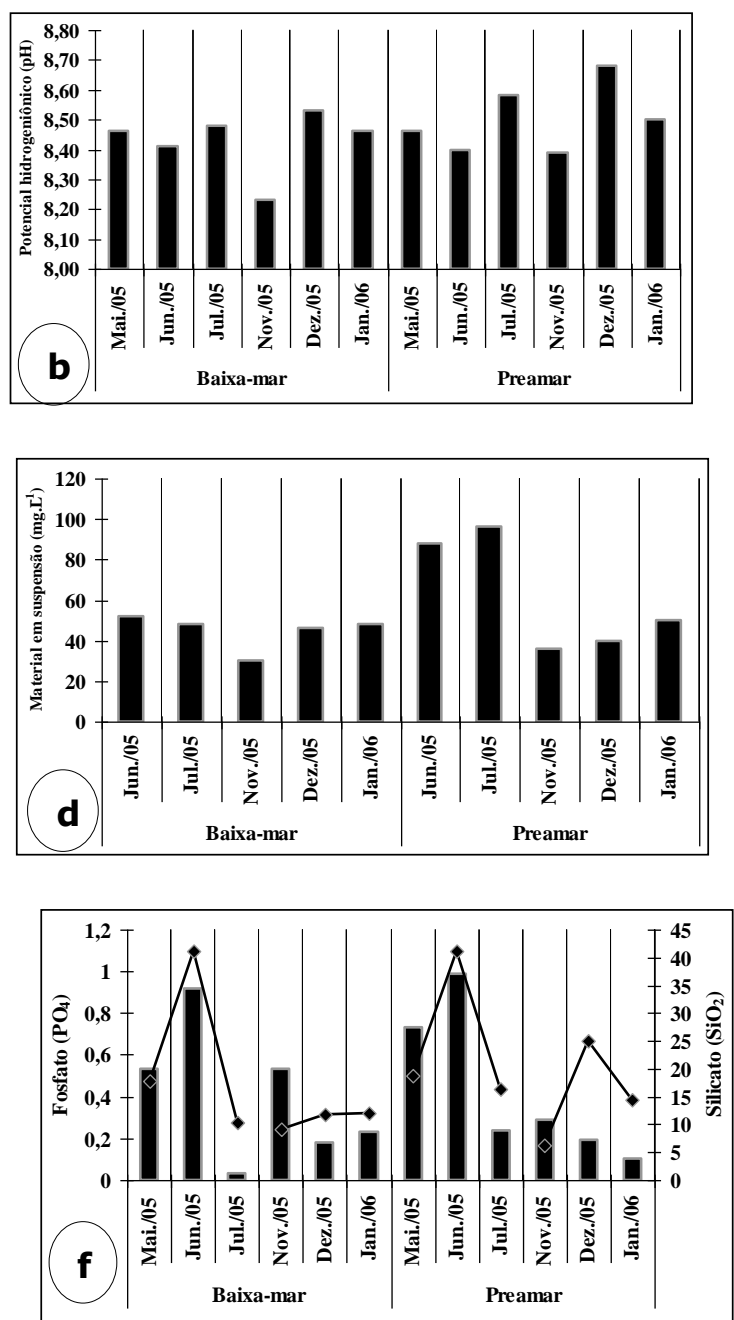

Figura 3 - Variação da temperatura da água (a-colunas), salinidade (a- linhas); potencial hidrogeniônico $(\mathrm{pH})(b)$; oxigênio dissolvido (c- colunas); taxa de saturação (clinhas); material em suspensão $(d)$; concentração dos íons nitrito (e-colunas); nitrato (e- linhas); fosfato ( $f$ - colunas) e silicato ( $f$ - linhas) na praia de Brasília Teimosa durante o período chuvoso (maio, jun. e jul./05) e período de estiagem (nov., dez/05 e jan./06).

\section{Parâmetros Biológicos:}

O teor de clorofila a total oscilou ao longo do ano apresentando um valor mínimo de $1,55 \mathrm{mg} \cdot \mathrm{m}^{-3}$ na baixa-mar de maio/05 e máximo de $17,06 \mathrm{mg} \cdot \mathrm{m}^{-3}$ em jan./06, também na baixa-mar. Apresentou uma média de 7,54, com uma amplitude de 15,51. Diferença significativa $(p=0,01)$ ocorreu quanto à variação do período sazonal, com maiores valores de clorofila a no período de estiagem (Fig. 4g). A densidade fitoplanctônica total variou de $60 \times 10^{3} \mathrm{cel} . \mathrm{L}^{-1}$ na baixa-mar de jun./05 e preamar de jul./05, a um máximo de $2.300 \times 10^{3} \mathrm{cel} . \mathrm{L}^{-1}$ na baixa-mar de dez./05. Apresentou uma média de $520 \times 10^{3} \mathrm{cel}^{-\mathrm{L}^{-1}}$ e uma amplitude de $2.240 \times 10^{3} \mathrm{cel}^{-1} \mathrm{~L}^{-1}$ (Fig. 4h). Não foi possível identificar diferenças sazonais $\mathrm{e}$ intertidais significativas $(p>0,05)$. 
FERREIRA, L. C. et al. Fitoplâncton como ferramenta de gestão ambiental na praia de Brasília Teimosa, Pernambuco, Nordeste do Brasil.

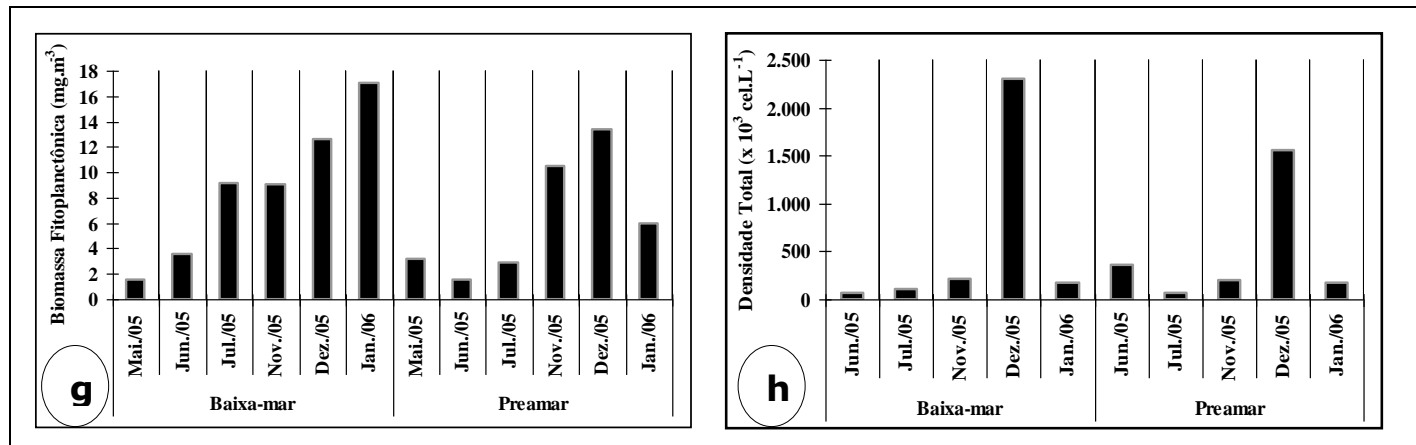

Figura 4 - Biomassa $(g)$ e densidade fitoplanctônica $(h)$ na praia de Brasília Teimosa durante o período chuvoso (maio, jun. e jul./05) e período de estiagem (nov., dez/05 e jan./06).

As diatomáceas apresentaram um valor mínimo de $10 \times 10^{3} \mathrm{cel} . \mathrm{L}^{-1}$ na preamar de jun./05 e máximo de $1.890 \times 10^{3} \mathrm{cel} . \mathrm{L}^{-1}$ na baixa-mar de dez./05, destacando-se as espécies Cerataulina pelagica (máximo de $450 \times 10^{3} \mathrm{cel} . \mathrm{L}^{-1}$ ), nos meses de nov./05 e dez./05, Cylindrotheca closterium (máximo de $400 \times 10^{3} \mathrm{cel} . \mathrm{L}^{-1}$ ) e Skeletonema costatum (máximo de $610 \times 10^{3} \mathrm{cel} . \mathrm{L}^{-1}$ ), em dez./05. Os fitoflagelados atingiram valor mínimo de $10 \times 10^{3} \mathrm{cel} . \mathrm{L}^{-1} \mathrm{em}$ jul./05 na preamar e máximo de $280 \times 10^{3} \mathrm{cel} . \mathrm{L}^{-1} \mathrm{em}$ dez./05, na baixa-mar. Os maiores valores dos grupos citados ocorreram no período de estiagem (Tab. 1).

Tabela 1 - Densidade fitoplanctônica (células por litro) dos principais grupos de microalgas identificadas na praia de Brasília Teimosa durante o período de estudo.

\begin{tabular}{|c|c|c|c|c|c|c|c|c|c|c|}
\hline & \multicolumn{2}{|c|}{ Jun./05 } & \multicolumn{2}{|c|}{ Jul./05 } & \multicolumn{2}{|c|}{ Nov./05 } & \multicolumn{2}{|c|}{ Dez./05 } & \multicolumn{2}{|c|}{ Jan./06 } \\
\hline & BM & PM & BM & PM & BM & PM & BM & PM & BM & PM \\
\hline Cianobactérias & ND & 320 & ND & ND & ND & 20 & 10 & 60 & 20 & 15 \\
\hline Euglenofíceas & 5 & ND & 20 & 5 & 10 & ND & 10 & 10 & ND & ND \\
\hline Dinoflagelados & ND & 5 & ND & 15 & 20 & ND & 10 & ND & ND & ND \\
\hline Fitoflagelados & 40 & 30 & 35 & 10 & 25 & 40 & 280 & 260 & 100 & 100 \\
\hline Diatomáceas & 15 & 10 & 45 & 30 & 160 & 140 & 1.890 & 1.210 & 45 & 60 \\
\hline Clorofíceas & ND & ND & ND & ND & ND & 5 & 100 & 10 & 5 & ND \\
\hline TOTAL & 60 & 365 & 100 & 60 & 215 & 205 & 2.300 & 1.550 & 170 & 175 \\
\hline Média & 20 & 91 & 33 & 15 & 54 & 51 & 383 & 310 & 43 & 58 \\
\hline Mínimo & 5 & 5 & 20 & 5 & 10 & 5 & 10 & 10 & 5 & 15 \\
\hline Máximo & 40 & 320 & 45 & 30 & 160 & 140 & 1.890 & 1.210 & 100 & 100 \\
\hline Amplitude & 35 & 315 & 25 & 25 & 150 & 135 & 1.880 & 1.200 & 95 & 85 \\
\hline
\end{tabular}

Durante o período estudado, os grupos das cianobactérias, euglenofíceas, dinoflagelados e clorofíceas foram pouco representativos, com exceção de um pico ocorrido no período chuvoso do gênero de cianobactéria Oscillatoria $\left(320 \times 10^{3} \mathrm{cel} . \mathrm{L}^{-1}\right)$ na preamar de jun./05.

\section{Composição Florística:}


FERREIRA, L. C. et al. Fitoplâncton como ferramenta de gestão ambiental na praia de Brasília Teimosa, Pernambuco, Nordeste do Brasil.

Foram inventariados 90 táxons, sendo 84 táxons infragenéricos (83 espécies e uma variedade) distribuídos em seis filos (Tabela 02). As Bacillariophyceae tiveram maior representatividade (68 spp.), seguida por Myzozoa (10 spp.), Chlorophyta (4 spp.), Euglenozoa (1 sp.) e Chrysophyta (1 sp.).

O índice de diversidade específica variou entre as categorias de baixa a alta diversidade. Dentre as amostras analisadas, $58,33 \%$ foram classificadas como alta diversidade enquanto $41,67 \%$, classificadas como baixa diversidade, apresentando uma distribuição com alta equitabilidade das amostras. A riqueza específica apresentou uma variação de 17 a 43 espécies identificadas, nas preamares de dez./05 e jun./05, respectivamente. Foi calculado um valor médio de 28, com uma amplitude de 26 (Fig. 5). Com relação às diferenças entre as marés e períodos sazonais, não foi possível observar significância $(p>0,05)$.

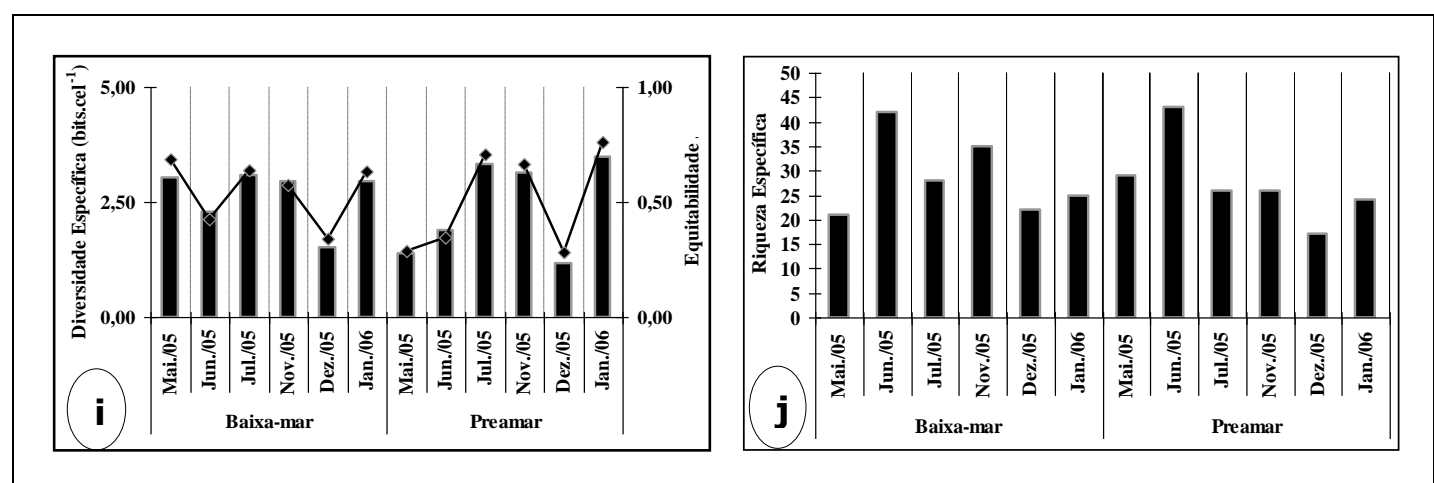

Figura 5 - Índice de diversidade específica ( $i$ - colunas), equitabilidade ( $i$-linhas) e Riqueza específica da comunidade microfitoplanctônica (j) em Brasília Teimosa durante o período chuvoso (maio, jun. e jul./05) e período de estiagem (nov., dez/05 e jan./06).

Foram dominantes as diatomáceas Asterionellopsis glacialis e Helicotheca tamesis. A. glacialis apresentou picos de abundância de $82,72 \%$ (972 células), 63,02\% (1.336 células) e $71,71 \%$ (1.778 células), durante as baixa-mares de maio/05 e jun.05, e preamar de jun./05, respectivamente, sendo característica do período chuvoso. A espécie $H$. tamesis apresentou dois picos de abundância, 66,54\% (25.166 células) e 79,40\% (8.040 células), durante o mês de dez./05 em ambas as marés, sendo característica do período de estiagem.

Quanto à frequência de ocorrência, 12 táxons foram classificados como muito frequentes, 14 como frequentes, 35 pouco frequentes e 29 registradas como esporádicas. Os táxons identificados foram distribuídos nas categorias ecológicas: planctônico marinho nerítico e oceânico, ticoplanctônico, estuarino e dulciaquícola (Tab. 2). As espécies marinhas representam a maior parcela do fitoplâncton, constituindo 90,91\% das espécies identificadas. 
FERREIRA, L. C. et al. Fitoplâncton como ferramenta de gestão ambiental na praia de Brasília Teimosa, Pernambuco, Nordeste do Brasil.

Tabela 02 - Sinopse, frequência de ocorrência e distribuição ecológica dos táxons identificados em Brasília Teimosa, durante o período chuvoso (maio, jun. e jul./05) e período de estiagem (nov., dez/05 e jan./06).

\begin{tabular}{|c|c|c|c|c|c|}
\hline Táxon & FO & $\overline{\mathrm{DE}}$ & Táxon & FO & $\overline{\mathrm{DE}}$ \\
\hline CYANOBACTERIA & & & Fragilaria capucina Desmazières & $\mathrm{F}$ & $\mathrm{T}$ \\
\hline Oscillatoria sp. & E & -- & Fragilaria sp. & $\mathrm{E}$ & --- \\
\hline EUGLENOZOA & & & Grammatophora marina (Lyngbye) & PF & $\mathrm{T}$ \\
\hline Euglena sp. & $\mathrm{PF}$ & --- & Guinardia flaccida (Castracane) $\mathrm{H}$. & $\mathrm{E}$ & $\mathrm{N}$ \\
\hline MYZOZOA & & & Guinardia striata (Stolterfoth) Hasle & $\mathrm{F}$ & $\mathrm{N}$ \\
\hline Ceratium furca (Ehr.) Clap. e & E & $\mathrm{N}$ & Helicotheca tamesis (Shrubsole) & MF & $\mathrm{N}$ \\
\hline Ceratium spp. & $\mathrm{E}$ & --- & Hemiaulus membranaceus Cleve & $\mathrm{E}$ & 0 \\
\hline Protoperidinium venustum & E & 0 & Leptocylindrus danicus Cleve & $\mathrm{F}$ & $\mathrm{N}$ \\
\hline Protoperidinium conicum (Gran) & $\mathrm{PF}$ & 0 & Licmophora abbreviata Agardh & MF & $\mathrm{T}$ \\
\hline Protoperidinium spp. & PF & -- & Licmophora sp. & $E$ & -- \\
\hline Pyrophacus horologicum Stein & PF & 0 & Lyrella lyra (Ehrenberg) Karayeva & $\mathrm{E}$ & $\mathrm{T}$ \\
\hline Pyrophacus steinii (Schiller) Wall & E & 0 & Melosira moniliformis (Müller) Agardh & PF & $\mathrm{T}$ \\
\hline Pyrophacus sp. & PF & -- & Melosira nummuloides Agardh & PF & $\mathrm{T}$ \\
\hline BACILLARIOPHYCEAE & & & Nitzschia longissima (Brèbisson) & $\mathrm{E}$ & $\mathrm{T}$ \\
\hline Achnanthes brevipes Agardh & PF & $\mathrm{T}$ & Odontella aurita (Lyngb.) Agardh & $\mathrm{PF}$ & $\mathrm{T}$ \\
\hline Achnanthes longipes Agardh & $\mathrm{E}$ & $\mathrm{T}$ & Odontella aurita var. obtusa (Kütz.) & PF & $\mathrm{T}$ \\
\hline Achnanthes sp. & $\mathrm{E}$ & --- & Odontella longicruris (Greville) Holan & PF & $\mathrm{N}$ \\
\hline Asterionellopsis glacialis & MF & $\mathrm{T}$ & Odontella mobiliensis (Bailey) Grunow & PF & $\mathrm{N}$ \\
\hline Bacillaria paxillifera (O. F. Müller) & $\mathrm{E}$ & 0 & Odontella tridens (Ehrenberg) & PF & $\mathrm{T}$ \\
\hline Bacteriastrum delicatulum Cleve & PF & 0 & Paralia sulcata (Ehrenberg) Cleve & MF & $\mathrm{T}$ \\
\hline Bacteriastrum hyalinum Lauder & PF & $\mathrm{N}$ & Plagiogramma sp. & MF & -- \\
\hline Bellerochea malleus (Brightwell) & $\mathrm{PF}$ & $\mathrm{N}$ & Pleurosigma sp. & PF & --- \\
\hline Biddulphia biddulphiana Smith & $\mathrm{F}$ & $\mathrm{T}$ & Podocystis adriatica Kützing & $\mathrm{PF}$ & $\mathrm{T}$ \\
\hline Biddulphia dubia (Brightwell) Cleve & E & $\mathrm{T}$ & Proboscia alata (Brightwell) Sundström & PF & 0 \\
\hline Campyloneis grevillei (Smith) & E & $\mathrm{T}$ & Psammodictyon sp. & $\mathrm{E}$ & -- \\
\hline Cerataulina pelagica (Cleve) & $\mathrm{F}$ & $\mathrm{N}$ & Pseudo-nitzschia sp. & $\mathrm{F}$ & --- \\
\hline Cerataulus turgidus Ehrenberg & E & $\mathrm{T}$ & Rhabdonema adriaticum Kutzing & PF & $\mathrm{T}$ \\
\hline Chaetoceros affinis Lauder & $\mathrm{F}$ & 0 & Rhizosolenia setigera Brightwell & $\mathrm{F}$ & 0 \\
\hline Chaetoceros atlanticus Cleve & $\mathrm{PF}$ & 0 & Rhizosolenia styliformis Brightwell & PF & 0 \\
\hline Chaetoceros brevis Schütt & PF & $\mathrm{N}$ & Stauropsis membranacea (Cleve) & $\mathrm{PF}$ & $\mathrm{N}$ \\
\hline Chaetoceros compressus Lauder & PF & $\mathrm{N}$ & Surirella sp. & $E$ & --- \\
\hline Chaetoceros curvisetus Cleve & MF & $\mathrm{N}$ & Synedra gailloni (Bory) Erhenberg & PF & ES \\
\hline Chaetoceros diversus Cleve & E & $\mathrm{N}$ & Thalassiosira subtilis (Ostenfeld) Gran. & $\mathrm{E}$ & 0 \\
\hline Chaetoceros lorenzianus Grunow & MF & $\mathrm{N}$ & Thalassionema frauenfeldii Grunow & $\mathrm{PF}$ & 0 \\
\hline Chaetoceros peruvianus & PF & 0 & Thalassionema nitzschioides Grunow & $\mathrm{F}$ & 0 \\
\hline Chaetoceros sp. & $\mathrm{F}$ & -- & Triceratium antediluvianum (Ehr.) & PF & $\mathrm{T}$ \\
\hline Chaetoceros subtilis Cleve & $\mathrm{PF}$ & ES & Triceratium pentacrinus Ehrenberg & PF & $\mathrm{T}$ \\
\hline Climacosphenia moniligera & $\mathrm{F}$ & $\mathrm{T}$ & CHRYSOPHYTA & & \\
\hline Corethron hystrix Hensen & $\mathrm{PF}$ & 0 & Dictyocha fibula Ehrenberg & $\mathrm{E}$ & 0 \\
\hline Coscinodiscus centralis Ehrenberg & $\mathrm{F}$ & 0 & CHLOROPHYTA & & \\
\hline Coscinodiscus spp. & PF & -- & Cylindrocapsa conferta W. West & $E$ & $\mathrm{D}$ \\
\hline Cylindrotheca closterium (Ehr.) & MF & $\mathrm{T}$ & Dictyosphaerium sp. & $\mathrm{E}$ & --- \\
\hline Dactyliosolen fragilissimus & $\mathrm{F}$ & $\mathrm{N}$ & Scenedesmus bicaudatus (Hansgirg) & $\mathrm{E}$ & $\mathrm{D}$ \\
\hline Entomoneis alata Ehrenberg & MF & $\mathrm{T}$ & Scenedesmus quadricauda (Turpin) & PF & D \\
\hline
\end{tabular}

\section{DISCUSSÃO}

Para o sistema aquático, a temperatura da água e a salinidade atuam como uma barreira ecológica, ou seja, muitos organismos encontrados em determinadas regiões geográficas só sobrevirão caso a salinidade e a temperatura local favoreça o seu desenvolvimento. Dessa forma, Fraga (1972), demonstra que a salinidade influência nas respostas funcionais, tais como crescimento, sobrevivência, reprodução e metabolismo dos organismos aquáticos. 
FERREIRA, L. C. et al. Fitoplâncton como ferramenta de gestão ambiental na praia de Brasília Teimosa, Pernambuco, Nordeste do Brasil.

No ambiente estudado, a salinidade e a temperatura da água tiveram pouca variação, sem privilegiar nenhum período característico do ano, comportamento considerado normal na zona costeira tropical.

As variações no regime pluviométrico e movimento gerado pelas ondas e marés exercem alterações na dinâmica de Brasília Teimosa, assim como a redução da transparência da água e da salinidade, provocada durante os meses de maior pluviosidade. Estas alterações acarretam um aporte mais intenso dos rios, carreando maior teor de nutrientes e materiais em suspensão, promovendo assim, um aumento do nível de mistura da água e diminuição da zona fótica.

Estas características inibem o desenvolvimento do fitoplâncton nesse período e incrementam as condições eutróficas do ambiente, fato registrado em outras áreas do Norte (PAIVA; ESKINAZI-LEÇA, 1990) e Nordeste do Brasil (ESKINAZI-LEÇA et al., 1997; PEREIRA et al., 2005, SANTIAGO et al., 2010).

Estes fatores físico-químicos contribuem para a distribuição espacial e temporal de organismos fitoplanctônicos, afetando também os teores de clorofila a (PHLIPS et al., 2002). A variação espaço-temporal da biomassa (clorofila a) pode proporcionar informações sobre o estado fisiológico do fitoplâncton, quantidade de células fitoplanctônicas e, indiretamente, sobre a composição taxonômica de uma população (MARGALEF, 1991).

No ambiente estudado, observou-se que a biomassa, juntamente com a densidade, foi influenciada diretamente pela hidrodinâmica, que faz com que haja uma maior ressuspensão dos sedimentos disponibilizando nutrientes para a coluna d'água (ODEBRECHT; GARCIA, 1998; VIDEAU et al., 1998; PERISSINOTTO et al., 2002).

Os indicadores desse forte hidrodinamismo foram: o oxigênio dissolvido (manteve-se supersaturado durante todo o período de estudo), o material em suspensão (elevado no período chuvoso) e a ocorrência de grande número de espécies ticoplanctônicas.

$\mathrm{Na}$ presença de ressurgências e ondas, geralmente a acumulação da biomassa fitoplanctônica é controlada pela disponibilidade de nitrogênio e fósforo, que são os fatores mais importantes na definição da estrutura da comunidade e dominância de determinadas espécies (KUDELA; DUGDALE, 2000).

De uma perspectiva sazonal, maiores teores de nutrientes foram encontrados no período chuvoso, como esperado, por haver maior interferência do aporte de água continental. As concentrações dos componentes inorgânicos caracterizam a praia de Brasília Teimosa apresentando-se em concentrações normais para esses ambientes com valores típicos de áreas costeiras, demonstrando uma eutrofização relacionada ao nitrato no período chuvoso.

O fitoplâncton, por constituir o primeiro elo da teia trófica nos ambientes aquáticos, necessita dos nutrientes para a fotossíntese e sua estrutura, variabilidade ecológica e diversidade são fundamentais para o funcionamento desses ecossistemas (GARIBOTTI et al., 2005). As diatomáceas contribuíram como as microalgas mais representativas, destacando-se as espécies: Asterionellopsis glacialis, Chaetoceros lorenzianus, Cylindrotheca closterium, Helicotheca tamesis, Licmophora abbreviata e Skeletonema costatum.

Sob o aspecto da diversidade específica, a praia de Brasília Teimosa apresenta alta diversidade, onde as amostras consideradas de baixa diversidade estiveram relacionadas à dominância das espécies Asterionellopsis glacialis e Helicotheca tamesis, consideradas oportunistas (r-estrategistas). Essas espécies apresentam uma alternância relacionada à sazonalidade, onde Helicotheca tamesis obteve destaque durante o período de estiagem e Asterionellopsis glacialis, no período chuvoso.

Segundo Rörig et al. (2003), a diminuição sazonal na abundância da diatomácea Asterionellopsis glacialis está relacionada à existência de um ciclo onde as células (e cistos) migram do sedimento para a coluna d'água, para a zona de surfe, retornando ao sedimento em condições calmas, implicando que a espécie possui estágios bênticos durante seu ciclo de vida, apesar de ser classificada como uma espécie tipicamente planctônica. 
FERREIRA, L. C. et al. Fitoplâncton como ferramenta de gestão ambiental na praia de Brasília Teimosa, Pernambuco, Nordeste do Brasil.

A alta diversidade específica encontrada em Brasília Teimosa deve-se à heterogeneidade do ambiente estudado, o qual recebe influência dos fluxos marinho e limnético, ligados diretamente aos recifes, estuário da bacia do Pina e Porto do Recife, condicionando desta maneira o maior aparecimento de espécies de diversas origens.

Segundo Nunes et al. (2005), a análise ambiental de Brasília Teimosa (utilizando "checklists") concluiu que a área estudada está fortemente impactada, onde o único indicador que aumentou foi o turismo, talvez pelo fato do bairro apresentar nova obra urbanística que dá acesso aos locais de interesse de recreação (Iate Clube do Recife, Bar do Dique, Casa de Banho e Parque das Esculturas de Brennand).

Esses impactos também puderam ser corroborados através da identificação de espécies bioindicadoras de águas em estado avançado de eutrofização, relativas aos gêneros: Oscillatoria (Cyanobacteria), Scenedesmus (Chlorophyta), Euglena (Euglenozoa), Synedra, Pleurosigma, Pseudo-nitzschia e Skeletonema costatum (Bacillariophyceae). Estes organismos também indicam um aporte de água doce e salobra relativas às águas da bacia do Pina e Porto de Recife.

Na praia de Brasília Teimosa a influência das atividades humanas é significativa, com mudanças na composição, densidade do fitoplâncton e biomassa (clorofila a), cujas análises revelaram a presença de indicadores de águas eutrofizadas, evidenciando um possível comprometimento da sanidade ambiental relacionado ao período de estiagem (período de maior exploração turística-recreativa).

\section{REFERÊNCIAS BIBLIOGRÁFICAS}

ARAGÃO, J.O.R. A influência dos oceanos Pacífico e Atlântico na dinâmica do tempo e do clima do Nordeste do Brasil. In: ESKINAZI-LEÇA, E.; NEUMANN-LEITÃO, S.; COSTA, M.F. (Orgs.). Oceanografia: Um cenário tropical. Recife: Bagaço, 2004. p. 287-317.

BRANCO, Â.C. Obras em Brasília Teimosa continuam. Jornal Gazeta Mercantil. São Paulo, 2004. Cadernos Regionais.

COUTINHO, P.N.; LIMA, A.T.O.; QUEIROZ, C.M.; FREIRE, G.S.S.; ALMEIDA, L.E.S.B.; MAIA, L.P.; MANSO, V.A.V.; BORBA, A.L.S.; MARTINS, M.H.A.; DUARTE, R.X. Estudos da erosão marinha nas praias de Piedade e de Candeias e no estuário de Barras das Jangadas. Relatório técnico, 1997.

ESKINAZI-LEÇA E.; SILVA-CUNHA, M.G.G.; KOENING, M.L.; MACEDO, S.J.; COSTA, K.M.P. (1997). Variação espacial e temporal do fitoplâncton na Plataforma Continental de Pernambuco - Brasil. Trabalhos Oceanográficos da Universidade Federal de Pernambuco, Recife, PE, v.25, p. 1-16, 1997. Disponível em:

<http://www.revista.ufpe.br/tropicaloceanography/volumes/volume_40_2_2012.htm>. Acesso em: 20 de julho de 2013.

FERRARIO, M.; SAR, E.; SALA, S. Metodología básica para el estudio del fitoplancton con especial referencia a las diatomáceas. In: ALVEAR, K.; FERRARIO, M.; OLIVEIRA-FILHO, E.C.; SAR, E. (Eds.). Manual de métodos ficológicos. Chile: Universidad de Concepción, 1995, p.1-24.

FRAGA, F. (1972). El água marina. In: CASTELLI, J. Ecologia marina. Caracas: Fundación La Salle de Ciencias Naturales, 1972. p. 67-99.

GARIBOTTI, I.A.; VERNET, M.; SMITH, R.S.; FERRARIO, M. E. Interannual variability in the distribution of the phytoplankton standing stock across the seasonal sea-ice zone west of the Antarctic Peninsula. Journal of Plankton Research, v.27, n.8, 2005p.825-843.

GRASSHOFF, K.; EHRARDT, M.; KREMELING, K. (1983). Methods of sea water analysis. 2 ed. New York: Verlag Chemie, 1983. 317p.

HASLE, G.R. The inverted-microscope methods. In: Sournia, A. (Ed.). Phytoplankton

manual. Paris: UNESCO, 1978. p. 88-96. 
FERREIRA, L. C. et al. Fitoplâncton como ferramenta de gestão ambiental na praia de Brasília Teimosa, Pernambuco, Nordeste do Brasil.

KUDELA, R.M.; DUGDALE, R.C. Nutrient regulation of phytoplankton productivity in Monterey Bay, California. Deep-Sea Research, v.47, p.1023-1053, 2000.

LOBO, E.; LEIGHTON, G. Estructuras comunitarias de las fitocenosia planctónicas de los sistemas de desembocaduras de ríos y esteros de la zona central de Chile. Revista Biología Marina, v.22, p.1-29, 1986.

MARGALEF, R. (1991). Ecologia. 7 ed. Barcelona: Omega, 1991, 951p.

MARGALEF, R. Les types biologiques de phytoplankton consideres comme des alternatives de survie dans um millieu instable. Ocoeanologia Acta, v.1, n.4, 1978. p.493-509.

MELO, U.; SUMMERHAYES, C.P.; TONER, L.G.. Metodologia para o estudo do material em suspensão na água do mar. Boletim Técnico da Petrobrás, Rio de Janeiro, v.18, n.3/4, 1975, p.115.

NEWELL, G.H.; NEWELL, R. Marine plankton: a practical guide. London: Hutchinson Educat, 1963. 221p.

NUNES, F.S.; PORTO NETO, F.F.; NEUMANN-LEITÃO, S. (2005). Condições Ambientais na Orla de Brasília Teimosa, Recife - Pernambuco: Re-Urbanização e sua Influência nos Recifes

Costeiros. In: Anais... II Congresso Brasileiro de Oceanografia, Vitória, 2005.

ODEBRECHT, C.; GARCIA, V.M.T. (1998). Fitoplâncton. In: SEELIGER, U.; ODEBRECHT, C.; CASTELLO, J.P. Os ecossistemas costeiro marinho do extremo sul do Brasil. Rio Grande: Ecoscientia, 1998. p.117-121.

PAIVA, R.S.; ESKINAZI-LEÇA, E. (1990). Variação da densidade (células/litro) do fitoplâncton da baía do Guajará (Pará-Brasil). In: Anais... Encontro Brasileiro de Plâncton 4, p. 123-138, 1990. Recife.

PEREIRA, L.C.C.; JIMÉNEZ, J.A.; KOENING, M.L.; PORTO-NETO, F.F.; MEDEIROS, C.; COSTA, R.M. Effect of Coastline Properties and Wastewater on Plankton Composition and Distribution in a Stressed Environment on the North Coast of Olinda-PE (Brazil). Brazilian Archives of Biology and Technology, v.48, n.6, 2005, p.1013-1026.

PERISSINOTTO, R.; NOZAIS, C.; KIBIRIGE, I. Spatio-temporal dynamics of phytoplankton and microphytobenthos in a South African temporally-open estuary. Estuarine, Coastal and Shelf Science, v.55, 2002, p.47-58.

PHLIPS, J.E.; BADYLAK, S.; GRASSKOFF. Factors Affecting the Abundance of Phytoplankton in a Restricted Subtropical Lagoon, the Indian River Lagon. Estuarine, Coastal and Shelf Science, v.55, 2002, p.385-402.

QUEIROZ, J. Indicadores biológicos para avaliar a água. AGRO C \& T, ano 1, n.3, 2002.

RABELO, E. Brasília Teimosa. Edição Extra, Recife, ano 1, n.2, 1968, p.10.

ROLLNIC, M.; MEDEIROS, C. Circulation of the Coastal Waters off Boa Viagem, Piedade and Candeias Beaches-PE, Brazil. Journal of Coastal Research, n.39, 2006. p.648 - 650.

RÖRIG, L.R.; GARCIA, V.M.T. Accumulations of the surf-zone diatom Asterionellopsis glacialis (CASTRACANE) ROUND in Cassino Beach, Southern Brazil, and its Relationship with

Environmental Factors. Journal of Coastal Research, n.35, 2003, p.167-177.

RÖRIG, L.R; ALMEIDA, T.C.M.; GARCIA, V.M.T. Structure and succession of the surf-zone phytoplankton in casino beach, southern Brazil. Journal of Coastal Research, n.39, 2006, p.1246-1250.

SHANNON, L.E. A mathematical theory of communication. Bulletin of System Technology Journal, v.27, 1948. p.379-423.

STRICKLAND, J.D.H.; PARSONS, T.R. A practical handbook of seawater analysis. Bulletin Fisheries Research board of Canada, v.167, 1972. p.1-205.

UNESCO. International Oceanographic Table. Wormly: Optichrome, v. 2, 1973. 141p. 
FERREIRA, L. C. et al. Fitoplâncton como ferramenta de gestão ambiental na praia de Brasília Teimosa, Pernambuco, Nordeste do Brasil.

UTERMÖHL, H. Zur vervollkommnung der quantitativen Phytoplankton Methodik. Mitteilung Internationale Vereinigung für Theoretische Angewandte Limnologie, v.9, 1958. p.6872.

VIDEAU, C.; RYCAERT, M.; L'HELGUEN, S. Phytoplankton em baie de Seine. Influence du panache fluvial sur la production primaire. Oceanologia Acta, Paris, v.21, n.6, 1998. p.907921. 